\title{
Kronecker Product of matrices and their applications to self-adjoint two-point boundary value problems associated with first order matrix differential systems
}

\author{
Sriram Bhagavatula ${ }^{1}$, Dileep Durani Musa ${ }^{2}$, K.N. Murty ${ }^{3}$ \\ ${ }^{1}$ Cinnamon Bay Servicing Inc. \\ ${ }^{2}$ Infosys \\ ${ }^{3}$ Department of Applied Mathematics, Andhra University, Visakhapatnam, India, 530003
}

\begin{abstract}
:
In this paper, we shall be concerned with Kronecker product or Tensor product of matrices and develop their properties in a systematic way. The properties of the Kronecker product of matrices is used as a tool to establish existence and uniqueness of solutions to two-point boundary value problems associated with system of first order differential systems. A new approach is described to solve the Kronecker product linear systems and establish best least square solutions to the problem. Several interesting examples are given to highlight the importance of Kronecker product of matrices. We present adjoint boundary value problems and deduce a set of necessary and sufficient conditions for the Kronecker product boundary value problem to be self-adjoint.
\end{abstract}

\section{AMS (MOS): 34A06, 34A30, 15A06, 15A09, 34B14}

Key words: Kronecker product of Matrices, Two-point boundary value problem, Linear differential system, Fundamental Matrix, Green's Function, Self-adjoint boundary value problem.

\section{Introduction:}

Kronecker product boundary value problems play an important role in many branches of science and Engineering. Recently its importance gained momentum in Linear Algebra, Systems theory, Matrix Calculus and Boundary value problems. Motivation to the study of two and multi-point boundary value problems can be cited by physical examples such as Vibrating String problem or Heat Conduction problem that can be described by linear equations. Our paper is organized as follows: Section 2 presents properties of Kronecker product of Matrices and develop basic theory to apply to two-point boundary value problems systematically . We present our main results on two-point boundary value associated with two first order linear systems of differential equations in a single framework using the properties of Kronecker product of matrices. The results established in this section are based on the fact that the homogeneous Kronecker product linear boundary problem has only the trivial solution. We then present a set of necessary and sufficient conditions for the Kronecker product boundary value problem to be self-adjoint. Significant contributions on Kronecker product boundary value problems can be found in the following references [1,2].The behavior of solutions of Kronecker product system of differential / difference equations are discussed at length by Viswanadh, Murty, Yan Wu, Divya and Sriram [5,6,8-11,13,14]. $\Psi$ - Bounded solutions on time scale dynamical system are established in [3]. Further some of the properties of Kronecker product of matrices and applications to boundary value problems are established in the recent works of Murty, Viswanadh, Yan Wu, Sriram and Dileep. Differential and Difference equations are used as a tool to build mathematical models to Physical 
Biological and agriculture sciences can be found in the works of Randal H Cole, Viswanadh, Murty, Anand , Rompicherla and Yan Wu [2,4-14].

Definition 2.1: Let $\mathrm{A}$ be an ( $\mathrm{m} \mathrm{x} \mathrm{n)} \mathrm{matrix} \mathrm{and} \mathrm{B}$ be a ( $\mathrm{p} \mathrm{x} \mathrm{q)} \mathrm{matrix.} \mathrm{Then} \mathrm{their} \mathrm{Kronecker} \mathrm{product}$ $(A \otimes B)$ is defined as

$(A \otimes B)=\left(a_{i j} B\right)(i=1 \ldots m ; j=1,2, \ldots n)$, and is an $(m p \times n q)$ matrix.

Remark1: Instead of the definition given above some authors used the definition

$$
A \otimes B=\left(\begin{array}{cccc}
b_{11} A & b_{12} A & \ldots & b_{1 q} A \\
b_{21} A & b_{22} A & \ldots & b_{2 q} A \\
& \ldots & \\
b_{p 1} A & b_{p 2} A & \ldots & b_{p q} A
\end{array}\right)=b_{k l} A, k=1, \ldots, p ; l=1, \ldots, q
$$

Throughout this paper, we use the Definition 2.1 only.

In general,

$$
\begin{gathered}
(A \otimes B) \neq(B \otimes A) \text { and } I_{m} \otimes I_{n}=I_{m n}=I_{n} \otimes I_{m} \\
I_{2} \otimes I_{3}=\left(\begin{array}{ll}
1 & 0 \\
0 & 1
\end{array}\right) \otimes I_{3}=I_{5}
\end{gathered}
$$

and

$$
I_{3} \otimes I_{2}=\left(\begin{array}{lll}
1 & 0 & 0 \\
0 & 1 & 0 \\
0 & 0 & 1
\end{array}\right) I_{2}=I_{5}
$$

where $\mathrm{I}$ is a unit matrix and $I_{5}$ is a unit matrix of order $(5 \times 5)$.

Let $\mathrm{u}=(1,0)^{T}$ and $v=(0,1)^{T}$ be the standard base vectors in $R^{2}$ or in $T^{2}$.

Then

$$
\begin{aligned}
& (u \otimes u)=\left(\left(\begin{array}{l}
1 \\
0
\end{array}\right) \otimes\left(\begin{array}{l}
1 \\
0
\end{array}\right)\right)=\left(\begin{array}{l}
1 \\
0 \\
0 \\
0
\end{array}\right) \text { and }(u \otimes v)=\left(\left(\begin{array}{l}
1 \\
0
\end{array}\right) \otimes\left(\begin{array}{l}
0 \\
1
\end{array}\right)\right)=\left(\begin{array}{l}
0 \\
1 \\
0 \\
0
\end{array}\right) \\
& \text { Similarly, }(v \otimes u)=\left(\begin{array}{l}
0 \\
0 \\
1 \\
0
\end{array}\right) \text { and }(v \otimes v)=\left(\begin{array}{l}
0 \\
0 \\
0 \\
1
\end{array}\right) .
\end{aligned}
$$

We note that $\{(u \otimes u),(u \otimes v),(v \otimes u),(v \otimes v)\}$ is the standard base vectors in $R^{4}$ ( or in $\left.T^{4}\right)$. It may be noted that if $\mathrm{A}$ is an ( $\mathrm{n} \times \mathrm{n}$ ) matrix and $Z$ is a column matrix of order ( $\mathrm{n} X 1)$ then

$\left(A \otimes I_{n}\right) Z=\left(A Z \otimes I_{n}\right)=A Z$

$\left(I_{n} \otimes Z\right) A=(A \otimes Z)$

$A\left(I_{n} \otimes Z\right)=A \otimes Z$.

Example 2.1: Consider the normalized vectors in $T^{2}$

$u=\frac{1}{\sqrt{2}}\left(\begin{array}{l}1 \\ i\end{array}\right)$ and $v=\frac{1}{\sqrt{2}}\left(\begin{array}{c}1 \\ -i\end{array}\right)$ 
Then

$$
(u \otimes u)=\frac{1}{2}\left(\begin{array}{c}
1 \\
i \\
i \\
-1
\end{array}\right),(u \otimes v)=\frac{1}{2}\left(\begin{array}{c}
1 \\
-i \\
i \\
1
\end{array}\right),(v \otimes v)=\frac{1}{2}\left(\begin{array}{c}
1 \\
i \\
-i \\
1
\end{array}\right),(v \otimes v)=\frac{1}{2}\left(\begin{array}{c}
1 \\
-i \\
-i \\
-1
\end{array}\right) .
$$

Since $\|u\|=\|v\|=1$ and $(\mathrm{u}, \mathrm{v})=0$ and hence $\{(u, v)\}$ is an Orthonormal basis in the vector space $T$. Further $\{(u \otimes u),(u \otimes v),(v \otimes u),(v \otimes) v\}$ is an Orthonormal basis in the vector space $T^{4}$.

Example 2.2: Let $u=\left(\begin{array}{l}u 1 \\ u 2\end{array}\right)$ and $v=\left(\begin{array}{l}v 1 \\ v 2\end{array}\right)$.

Then

$$
\begin{aligned}
& u^{*} \otimes u=\left(\overline{u_{1}} \overline{u_{2}}\right) \otimes\left(\begin{array}{l}
u_{1} \\
u_{2}
\end{array}\right)=\left(\begin{array}{lll}
\overline{u_{1}} u_{1} & \overline{u_{1}} u_{2} \\
\overline{u_{2}} u_{1} & \overline{u_{2}} u_{2}
\end{array}\right) \\
& v^{*} \otimes v=\left(\overline{v_{1}} \overline{v_{2}}\right) \otimes\left(\begin{array}{l}
v_{1} \\
v_{2}
\end{array}\right)=\left(\begin{array}{ll}
\overline{v_{1}} v_{1} & \overline{v_{1}} v_{2} \\
\overline{v_{2}} v_{1} & \overline{v_{2}} v_{2}
\end{array}\right)
\end{aligned}
$$

$u^{*} \otimes v=\left(\overline{u_{1}} \overline{u_{2}}\right) \otimes\left(\begin{array}{l}v_{1} \\ v_{2}\end{array}\right)=\left(\begin{array}{ll}\overline{u_{1}} v_{1} & \overline{u_{1}} v_{2} \\ \overline{u_{2}} v_{1} & \overline{u_{2}} v_{2}\end{array}\right)$.

And

$$
v^{*} \otimes u=\left(\overline{v_{1}} \overline{v_{2}}\right) \otimes\left(\begin{array}{l}
u_{1} \\
u_{2}
\end{array}\right)=\left(\begin{array}{ll}
\overline{v_{1}} u_{1} & \overline{v_{1}} u_{2} \\
\overline{v_{2}} u_{1} & \overline{v_{2}} u_{2}
\end{array}\right)
$$

Thus, we find that

$$
\left(u^{*} \otimes v\right)=u^{*} \otimes v \text { and }\left(u^{*} \otimes u\right)=u u^{*}
$$

If $u=\frac{1}{\sqrt{2}}\left(\begin{array}{l}1 \\ 1\end{array}\right)$ and $v=\frac{1}{\sqrt{2}}\left(\begin{array}{c}1 \\ -1\end{array}\right)$,

Then

$\left(u^{*} \otimes u\right)=\frac{1}{2}\left(\begin{array}{ll}1 & 1 \\ 1 & 1\end{array}\right)$ and $\left(v^{*} \otimes v\right)=\frac{1}{2}\left(\begin{array}{cr}1 & -1 \\ -1 & 1\end{array}\right)$,

and consequently

$$
\left(u^{*} \otimes u\right)+\left(v^{*} \otimes v\right)=\left(\begin{array}{ll}
1 & 0 \\
0 & 1
\end{array}\right)=I_{2} \text {. Thus } u^{*} u+v^{*} v=I_{2}
$$

Definition 2.1: Let $\mathrm{A}$ be an $(\mathrm{m} \times \mathrm{n})$ matrix and $\mathrm{B}$ be an $(\mathrm{p} \times \mathrm{q})$ matrix. Then the symmetric tensor product is defined as

$A \otimes B=\frac{1}{2}((A \otimes B)+(B \otimes A))$.

We now list some of the basic properties of the Kronecker product of matrices.

Let $\mathrm{A}$ be an $(\mathrm{m} \times \mathrm{n})$ matrix and $\mathrm{B}$ be an $(\mathrm{p} \times \mathrm{q})$ matrix and $\mathrm{C}$ be an $(r \times \mathrm{s})$ matrix.

Then $(A \otimes B) \otimes C=A \otimes(B \otimes C)$.

Proof: $(A \otimes B)$ is an $(m p \times n q)$ matrix and $(A \otimes B) \otimes C$ is an (mpr x nqs) matrix. Similarly, $(B \otimes C)$ is an (pr x qs) matrix and $A \otimes(B \otimes C)$ also an (mpr x nqs) matrix and it can easily be shown that (ijk) ${ }^{\text {th }}$ element 
the component of $(A \otimes B) \otimes C$ is same as $A \otimes(B \otimes C)$. Hence Kronecker product of matrices satisfies the associative law. The Kronecker product of matrices obeys distributive law

$(A+B) \otimes(C+D)=(A \otimes C)+(A \otimes D)+(B \otimes C)+(B \otimes D)$.

We can easily prove the row rank and column rank are equal. Let $\rho(\mathrm{A})$ be the rank of $A$ and $\rho(B)$ to the rank of B. Then $\rho(A \otimes B)=\rho(A) \rho(B)$.

Let $\mathrm{A}=\left[\begin{array}{ll}0 & 1 \\ 2 & 0\end{array}\right]$ and $\mathrm{B}=\left[\begin{array}{ll}0 & 0 \\ 1 & 0\end{array}\right]$ then $\rho(\mathrm{A})=2$ and $\rho(\mathrm{B})=1$. Therefore $\rho(A \otimes B)=2$.

Note that

$(A \otimes B)^{T}=\left(A^{T} \otimes B^{T}\right),(\overline{A \otimes B})=\bar{A} \otimes \bar{B}$ and $(A \otimes B)^{*}=\left(A^{*} \otimes B^{*}\right)$.

If $\mathrm{A}$ and $\mathrm{B}$ are upper (lower) triangular matrices, then $(A \otimes B)$ is upper (lower) triangular matrix.

If $\mathrm{A}$ and $\mathrm{B}$ are diagonal matrices, then $(\mathrm{A} \otimes B)$ is also a diagonal matrix and if $(A \otimes B) \neq(0 \otimes 0)$ then $\mathrm{A}$ and $\mathrm{B}$ are diagonal matrices. If $\mathrm{A}$ and $\mathrm{B}$ are normal matrices, then $(A \otimes B)$ is a normal matrix.

If $\mathrm{A}$ and $\mathrm{B}$ are Hermitian matrices, then $\mathrm{A} \otimes B$ is an Hermitian matrix. For

$(A \otimes B)^{*}=\left(A^{*} \otimes B^{*}\right)=(A \otimes B)$.

If $A$ is an Hermitian matrix and $B$ is skew Hermitian matrix, then $(A \otimes B)$ is a skew-Hermitian matrix.

For

$(A \otimes B)^{*}=\left(A^{*} \otimes B^{*}\right)=(A \otimes-B)=-(A \otimes B)$.

If $\mathrm{A}$ and $\mathrm{B}$ are Skew - Hermitian matrices, then $(A \otimes B)$ is not a Skew - Hermitian matrix and is in fact Hermitian. For

$(A \otimes B)^{*}=\left(A^{*} \otimes B^{*}\right)=((-A) \otimes(-B))=(A \otimes B)$.

If $\mathrm{A}$ is an ( $\mathrm{mx} \mathrm{n}$ ) matrix then

$A^{k}=A^{k-1} \otimes A=A \otimes A^{K-1}$ for any $K \geq 1$.

In general,

$A^{k} \otimes A^{l}=A^{(K+l)}$ where $K \in N, l \in N$.

Theorem 3.3: Let $\mathrm{A}$ be an $(\mathrm{m} \mathrm{x} \mathrm{m})$ in invertible matrix and $\mathrm{B}$ be a $(\mathrm{k} \mathrm{x} \mathrm{k})$ invertible matrix. Then $(A \otimes B)^{-1}=\left(A^{-1} \otimes B^{-1}\right)$.

Proof: Since A and B are non-singular matrices, we have

$|A| \neq 0$ and $|B| \neq 0$ and

$\operatorname{det}(A \otimes B)=[\operatorname{det}(\mathrm{A})]^{m}[\operatorname{det}(\mathrm{B})]^{p}$ and so

$\operatorname{Det}(A \otimes B) \neq 0$ then $(A \otimes B)^{-1}$ exists and

$(A \otimes B)(A \otimes B)^{-1}=(A \otimes B)\left(A^{-1} \otimes B^{-1}\right)$

$$
\begin{aligned}
& =\left(A A^{-1} \otimes B B^{-1}\right) \\
& =I_{m} \otimes I_{p} \\
& =I_{\mathrm{mp}}
\end{aligned}
$$


Similarly

$$
\begin{aligned}
(A \otimes B)^{-1}(A \otimes B)= & \left(A^{-1} \otimes B^{-1}\right)(A \otimes B) \\
= & \left(A^{-1} A \otimes B^{-1} B\right) \\
= & I_{m} \otimes I_{p} \\
= & I_{\mathrm{mp} .}
\end{aligned}
$$

Hence $(A \otimes B)^{-1}=\left(A^{-1} \otimes B^{-1}\right)$.

Definition 2.2: Let $\mathrm{A}$ and $\mathrm{B}$ be similar matrices of order $(\mathrm{m} \mathrm{x} \mathrm{m})$. Then there exists a non-singular matrix $\mathrm{T}$ of order ( $\mathrm{m} \mathrm{x} \mathrm{m}$ ) such that $\mathrm{B}=T^{-1} A T$.

Note that if $\mathrm{A}$ and $\mathrm{B}$ are similar, then $(A \otimes A)$ and $(B \otimes B)$ are similar. If $\mathrm{A}$ be an $(\mathrm{m} \mathrm{x} \mathrm{m})$ matrix and $\mathrm{B}$ be an ( $\mathrm{n} \times \mathrm{n})$ matrix, then

$\operatorname{Sin}\left(A \otimes I_{n}+I_{m} \otimes B\right)=\operatorname{Sin}(A) \otimes \operatorname{Cos}(B)+\operatorname{Cos}(A) \otimes \operatorname{Sin}(B)$.

Let $\mathrm{A}$ and $\mathrm{B}$ be Idempotent matrices, then

$(A \otimes B)^{2}=(A \otimes B)(A \otimes B)=\left(A^{2} \otimes B^{2}\right)=\left(I_{m} \otimes I_{m}\right)$.

We now turn our attention to the eigenvalue problems.

Definition 3.3: A value of $\chi$ for which $|A-\lambda I|=0$ is called an eigenvalue of $A$ and corresponding non-zero vector $x$ such that $A x=\lambda x$ is called an eigenvector of $A$.

Theorem 3.4: Let $\mathrm{A}$ be an ( $\mathrm{x} \mathrm{n})$ matrix with eigenvalue $\chi$ and $\mathrm{x}$ be its non-zero eigenvector and $\mathrm{B}$ be $\mathrm{a}(\mathrm{p} \mathrm{x}$ p) matrix with eigenvalue $\mu$ and with corresponding non-zero eigenvector $\mu$. Then their Kronecker product $(A \otimes B)$ has the eigenvalue $\lambda \mu$ with the corresponding eigenvector (non-zero) $(x \otimes y)$.

Proof: From hypothesis, we have

$\mathrm{Ax}=\lambda x$ and $\mathrm{By}=\lambda y$.

Then $(\mathrm{Ax}) \otimes(B y)=(\lambda x \otimes \mu Y)$

$$
=\lambda \mu(x \otimes y) .
$$

Since $(\mathrm{Ax}) \otimes(B y)=(A \otimes B)(x \otimes y)$

$$
=\lambda \mu(x \otimes y) .
$$

This equation clearly shows the $(\lambda \mu)$ is an eigenvector of $(A \otimes B)$ with corresponding non-zero eigenvector $(x \otimes y)$

Example: Let $A=\left[\begin{array}{cc}1 & 0 \\ 0 & -1\end{array}\right]$ and $\left[\begin{array}{cc}0 & 1 \\ -1 & 0\end{array}\right]$.

Since the eigenvector of $\mathrm{A}$ are $1,-1$ and $\mathrm{B}$ are $1,-1$, we find that the eigenvalues $(A \otimes B)$ are $(1,1,-1,-1)$.

Theorem 3.4: Let $\lambda$ be an eigenvalue of $\mathrm{A}$ of order $(\mathrm{m} \mathrm{x} \mathrm{m})$ and $\mu$ be an eigenvalue of $\mathrm{B}$ of order $(\mathrm{n} \times \mathrm{n})$.

Then $(A \otimes B)$ has an eigenvalue $\lambda+\mu$.

Proof: Let $\mathrm{x}$ and $\mathrm{y}$ be eigenvectors of $\mathrm{A}$ and $\mathrm{B}$ with corresponding eigenvalues $\lambda$ and $\mu$ respectively. Then

$\left(A \otimes I_{n}+I_{m} \otimes B\right)(x \otimes y)=\left(A \otimes I_{n}\right)(x \otimes y)+\left(I_{m} \otimes B\right)(x \otimes y)$

$$
=(A x \otimes y)+(x \otimes B y)
$$




$$
\begin{aligned}
& =(\lambda x \otimes y)+(x \otimes \mu y) \\
& =\lambda(x \otimes Y)+\mu(x \otimes y) \\
& =(\lambda+\mu)(x \otimes y) .
\end{aligned}
$$

Thus, the proof of the theorem is complete.

Theorem 3.5: The eigenvalues of Hermitian matrix are all real.

Proof: Let $\mathrm{A}$ be ( $\mathrm{n} \mathrm{x} \mathrm{n})$ Hermitian matrix with eigenvalue $\lambda$ and corresponding non-zero eigenvector being $\mathrm{x}$, then we have

$$
\begin{aligned}
& A x=\lambda x \\
& \quad(A x)^{*}=(\lambda x)^{*}=\bar{\lambda} x^{*} \\
& A x=\lambda x \text { and } B y=\mu y .
\end{aligned}
$$

Consider

$(A x \otimes B y)=(A \otimes B)(x \otimes y)=\lambda \mu(x \otimes y)$

Or

$\left(x^{*} \otimes y^{*}\right)(A \otimes B)(x \otimes y)=\lambda \mu\left(x^{*} \otimes y^{*}\right)(x \otimes y)$.

Again

$[(\mathrm{A} \otimes B)(x \otimes y)]^{*}=\bar{\lambda} \bar{\mu}\left(x^{*} \otimes y^{*}\right)$

$\left(x^{*} \otimes y^{*}\right)\left(A^{*} \otimes B^{*}\right)=\bar{\lambda} \bar{\mu}\left(x^{*} \otimes y^{*}\right)$

$\left(x^{*} \otimes y^{*}\right)(A \otimes B)(x \otimes y)=\bar{\lambda} \bar{\mu}\left(x^{*} \otimes y^{*}\right)(x \otimes y)$

Equations (3.2) and (3.3) gives

$0=(\lambda \mu-\bar{\lambda} \bar{\mu})\left(x^{*} \otimes y^{*}\right)(x \otimes y)$

This implies $\lambda \mu-\bar{\lambda} \bar{\mu}=0$ or $\lambda \mu=\bar{\lambda} \bar{\mu}$.

Hence, eigenvalues of a Hermitian matrix are all real.

1. Adjoint Kronecker product Boundary value problem

In this section, we establish adjoint Kronecker product boundary value problem and deduce conditions under which a Kronecker product boundary value problem is self-adjoint. We consider

$L_{1} y=P_{1} y^{\prime}+Q_{1} y, a \leq t \leq b$

(4.1)

and

$L_{2} Z=P_{2} Z^{\prime}+Q_{2} Z, a \leq t \leq b$

where $P_{1}, P_{2}$ are square matrices of order $\mathrm{n}, P_{1}(t) \in C^{2}[\mathrm{a}, \mathrm{b}]$ And $P_{2}(t) t C^{2}[a, b]$ and $P 1$ and $P 2$ are non-singular matrices $Q_{1} \in C^{1}[a, b]$ and $Q \in C^{2}[a, b]$ are non-singular matrices of order.

Equations (4.1) and (4.2) can conveniently be recast as 


$$
\begin{aligned}
& L y=L_{1}(y \otimes z)=\left(P_{1} \otimes I_{n}\right)\left(I_{n} \otimes P_{2}\right)(y \otimes z)^{\prime}+\left(Q_{1} \otimes I_{n}+I_{n} \otimes Q_{2}\right)(y \otimes z) \\
& =\left(P_{1} \otimes P_{2}\right)(y \otimes z)^{\prime}+\left(Q_{1} \otimes Q_{2}\right)(y \otimes z) .
\end{aligned}
$$

The adjoint equation is of special significance in the theory of differential equations. This notion was originally introduced by Lagrange in connection with the problem of finding an integrating factor. Here also we introduce in the same point of view. i.e., problem of finding an $\left(n^{2} \times 1\right)$ vector such that the inner product is a perfect derivative.

Let $L u=\left(P_{1} \otimes P_{2}\right) u^{\prime}+\left(Q_{1} \otimes Q_{2}\right) u$,

When $\mathrm{L}=L_{1} \otimes L_{2}, u=(\mathrm{Y} \otimes \mathrm{Z})$. Taking the inner product with the vector $\mathrm{V}$ of order $\left(n^{2} \times 1\right)$, we get

$$
\begin{aligned}
v^{*} L u & =v^{*}\left(P_{1} \otimes P_{2}\right) u^{\prime}+v^{*}\left(Q_{1} \otimes Q_{2}\right)(u) \\
& =\left(v^{*}\left(P_{1} \otimes P_{2}\right) u\right)^{\prime}-\left(v^{*}\left(P_{1} \otimes P_{2}\right)\right)^{\prime} u+\left(v^{*}\left(Q_{1} \otimes Q_{2}\right) u\right)
\end{aligned}
$$

$v^{*} L u-L^{*} v u=\left[v^{*}\left(P_{1} \otimes P_{2}\right) u\right]^{\prime}$

Where $L^{*} v=-\left[v^{*}\left(P_{1} \otimes P_{2}\right)^{*}\right]^{\prime}+v^{*}\left(Q_{1} \otimes Q_{2}\right)$

is known as the adjoint equation of (4.3), where

$$
L^{*} v=\left(L_{1}^{*} \otimes L_{2}^{*}\right) v=\left(L_{1}^{*} \otimes L_{2}^{*}\right)\left(v_{1} \otimes v_{2}\right)^{*}
$$

The operator $L$ is said to be self-adjoint, if it is identical with the operator $L^{*}$.

$\left(P_{1} \otimes P_{2}\right)^{*}=-\left(P_{1} \otimes P_{2}\right)$

and

$\left(Q_{1} \otimes Q_{2}\right)-\left(Q_{1} \otimes Q_{2}\right)^{*}=\left(P_{1} \otimes P_{2}\right)^{\prime}$

Note that $\left(P_{1} \otimes P_{2}\right)$ is a skew-hermetian matrix if either $\mathrm{P}_{1}$ or $\mathrm{P}_{2}$ is skew-hermitian. If $\mathrm{P}_{1}$ and $\mathrm{P}_{2}$ are skewhermitian, then $\left(P_{1} \otimes P_{2}\right)$ becomes a Hermitian matrix.

We now turn our attention to the kronecker product boundary value problem.

$L_{1} y=P_{1} y^{\prime}+Q_{1} y, a \leq t \leq b$

$M_{1} y(a)+N_{1} y(b)=0$

and

$L_{2} Z=P_{2} Z^{\prime}+Q_{2} Z, a \leq t \leq b$

$M_{2} Z(a)+N_{2} Z(b)=0$.

(4.6) and (4.7) can be embedded as

$L x=\left(P_{1} \otimes P_{2}\right) x^{\prime}+\left(Q_{1} \otimes Q_{2}\right) x, \quad a \leq t \leq b$

$$
\left(M_{1} \otimes M_{2}\right) x(a)+\left(N_{1} \otimes N_{2}\right) x(b)=0 .
$$

Where $x=\left(\begin{array}{l}y \\ z\end{array}\right)$. Note that $\mathrm{M}_{1}, \mathrm{M}_{2}, \mathrm{~N}_{1}$ and $\mathrm{N}_{2}$ connect square matrices of order $\mathrm{n}$. 
The boundary condition matrix in (4.8) can be put as

$\left(\left(M_{1} \otimes M_{2}\right) \quad\left(N_{1} \otimes N_{2}\right)\right)\left(\begin{array}{l}x(a) \\ x(b)\end{array}\right)=0$

$\mathrm{R} \mathrm{b} x=0$

Where $\mathrm{R}$ is an $\left(n^{2} \times 2 n^{2}\right)$ matrix. Since $\mathrm{R}$ is an $\left(\left(n^{2} \times 2 n^{2}\right)\right)$ of matrix of rank $n^{2}$, there exists a matrix $\mathrm{U}$ with $\left(2 n^{2}-n^{2}\right)=n^{2}$ linearly independent columns such that $\mathrm{R} \mathrm{U}=0$. The adjoint boundary value problem is given by

$L^{*} v=-\left[v^{*}\left(P_{1} \otimes P_{2}\right)\right]^{\prime}+v^{*}\left(Q_{1} \otimes Q_{2}\right)$

$$
U^{*} B^{*} b z=0
$$

The boundary value problem is said to be self-adjoint if it is identical with the adjoint boundary value problem (4.1). In this case

$U^{*} B^{*}=R T$,

When $\mathrm{T}$ is non-singular. On elimination $\mathrm{T}$, we get

$\left(M_{1} \otimes M_{2}\right)\left(P_{1} \otimes P_{2}\right)^{-1}(a)\left(M_{1}^{*} \otimes M_{2}^{*}\right)=\left(N_{1} \otimes N_{2}\right)\left(P_{1} \otimes P_{2}\right)^{-1}(b)\left(N 1^{*} \otimes N 2^{*}\right)$.

Thus the necessary and sufficient condition for the boundary value problem to be self-adjoint is

$\left(P_{1} \otimes P_{2}\right)^{*}=-\left(P_{1} \otimes P_{2}\right)$

$\left(Q_{1} \otimes Q_{2}\right)-\left(Q_{1} \otimes Q_{2}\right)^{*}=\left(P_{1} \otimes P_{2}\right)^{\prime}$

and

$\left(M_{1} \otimes M_{2}\right)\left(P_{1}^{-1} \otimes P_{2}^{-1}\right)(a)\left(M_{1}^{*} \otimes M_{2}^{*}\right)=\left(N_{1} \otimes N_{2}\right)\left(P_{1}^{-1} \otimes P_{2}^{-1}\right)(b)\left(N_{1}^{*} \otimes N_{2}^{*}\right)$.

\section{References}

1. E.A. Coddington and N. Levinson, Theory of ordinary differential equations, Mc Graw-hill, New York, 1955.

2. Randal H. Cole, Theory of ordinary differential equations, Application Century Craft, 1967.

3. Kasi Viswanadh V. Kanuri, R. Suryanarayana, K. N. Murty, Existence of $\Psi$-bounded solutions for linear differential systems on time scales, Journal of Mathematics and Computer Science, 20 (2020), no. 1, 1--13..

4. Kasi Viswanadh V Kanuri, Existence Of $\Psi$-Bounded Solutions For Fuzzy Dynamical Systems On Time Scales, International Journal of Scientific \& Engineering Research, 2020, Vol. 11, No. 5, 613-624.

5. Murty, K. N., Andreou, S., Viswanadh, K. V. K., Qualitative properties of general first order matrix difference systems. Nonlinear Studies, 16(2009), no. 4, 359--370.

6. K. N. Murty, V. V. S. S. S. Balaram, K.V. K. Viswanadh, "Solution of Kronecker Product Initial Value Problems Associated with First Order Difference System via Tensor-based Hardness of the Shortest Vector Problem", Electronic Modeling, vol. 6, p: 19--33 (2008).

7. Kasi Viswanadh V Kanuri, Y. Wu, K.N. Murty, "Existence of $(\Phi \otimes \Psi)$ bounded solution of linear first order Kronecker product of system of differential equations", International Journal of Science and Engineering Research, 2020, Vol 11, No. 6, P: 156--163.

8. Kanuri, K. V. V., Bhagavathula, S., \& Murty, K. (2020). Stability Analysis of Linear Sylvester System of First Order Differential Equations. International Journal of Engineering and Computer Science, 9(11), 25252--25259. 
9. Divya L Nethi, Yan Wu, Sriram Bhagavathula, K.N Murty, Cryptographic Algorithm to Find Best Least Square Solutions of Kronecker Product Boundary Value Problems, 2021, Vol. 8, No. 1, pp: 1970--1980.

10. K.N. Murty, Yan Wu, Viswanadh V. Kanuri, "Metrics that suit for dichotomy, well conditioning of object oriented design", Nonlinear Studies, Vol 18, No: 4, P: $621--637$ (2013).

11. Dileep Durani Musa, Yan Wu, Divya L Nethi, "Two-point Boundary value problems associated with first order nonhomogeneous Sylvester systems of differential equations", International Journal of recent scientific research, Vol 12, No. 8, 2021

12. Lakshmi N.V., Madhu J., Dileep Durani, M., "On the Kronecker Product of Matrices and Their Applications To Linear Systems Via Modified QR-Algorithm”, International Journal of Engineering and Computer Science, Vol 10, No 6, PP 25352-25359, 2021.

13. Y. Wu, D.L. Nethi, K.N.Murty, Initial Value Problems Associated With First Order Fuzzy Difference System -Existence And Uniqueness, International Journal of Recent Scientific Research. 11 (2020), no. 3, 37846--37848.

14. Kasi Viswanadh V. Kanuri, K. N. Murty, "Three-Point boundary value problems associated with first order matrix difference system-existence and uniqueness via shortest and closest Lattice vector methods", Journal of Nonlinear Sciences and Applications, Volume 12, Issue 11, (2019) 720-727. 